\title{
Inverse Agonist of Estrogen-Related Receptor $\gamma$ Enhances Sodium Iodide Symporter Function Through Mitogen-Activated Protein Kinase Signaling in Anaplastic Thyroid Cancer Cells
}

\author{
Thoudam Debraj Singh ${ }^{1}$, Shin Young Jeong ${ }^{1}$, Sang-Woo Lee ${ }^{1}$, Jeoung-Hee $\mathrm{Ha}^{2}$, In-Kyu Lee ${ }^{3}$, Seong Heon Kim ${ }^{4}$, \\ Jina Kim ${ }^{4}$, Sung Jin Cho ${ }^{4}$, Byeong-Cheol Ahn ${ }^{1}$, Jaetae Lee ${ }^{1,4}$, and Young Hyun Jeon ${ }^{1,5}$ \\ ${ }^{I}$ Department of Nuclear Medicine, School of Medicine, Kyungpook National University, Daegu, Korea; ${ }^{2}$ Department of \\ Pharmacology, School of Medicine, Kyungpook National University, Daegu, Korea; ${ }^{3}$ Department of Internal Medicine, School of \\ Medicine, Kyungpook National University, Daegu, Korea; ${ }^{4}$ New Drug Development Center, Daegu-Gyeongbuk Medical Innovation \\ Foundation, Daegu, Korea; and ${ }^{5}$ Leading-Edge Research Center for Drug Discovery and Development for Diabetes and Metabolic \\ Disease, Kyungpook National University Hospital, Daegu, Korea
}

Anaplastic thyroid cancer (ATC), a rare thyroid cancer with poor prognosis, is associated with insufficient function of the sodium iodide symporter (NIS). Estrogen-related receptor y (ERRy) is a member of the orphan nuclear receptors with important functions in cell development and homeostasis. However, there are no reports that demonstrate whether ERRy is related to NIS function. Here, we evaluated the role of ERRy in the regulation of NIS function in ATC cells using GSK5182, an inverse agonist of ERRy. Methods: Two ATC cell lines, BHT-101 and CAL62, were incubated with GSK5182 at various time points and doses. The NIS function in the ATC cells was serially assessed by their uptake of radioiodine. The effects of GSK5182 on ERRy and the mitogen-activated protein (MAP) kinase pathway, as well as on NIS protein, were evaluated by immunoblot assay. To examine whether the GSK5182-induced NIS functional activity can be affected by inhibition of the MAP kinase pathway, the MAP kinase activity and levels of radioiodine uptake were determined after application of a mitogen-activated protein kinase kinase (MEK) inhibitor to GSK5182treated cells. Finally, the cytotoxic effect of ${ }^{131}$ I was determined by clonogenic assay. Results: Treatment with GSK5182 resulted in doseand time-dependent increases in iodide uptake in ATC cells, which were accompanied by both the downregulation of ERRy protein and the activation of extracellular signal-regulated kinase (ERK) 1/2. Both the increased radioiodine uptake and ERK1/2 activation of ATC cells were completely inhibited by the specific MEK inhibitor. GSK5182 treatment enhanced the membrane localization of NIS in both ATC cell lines. Accordingly, preexposure to GSK5182 enhanced the cytotoxic effects of ${ }^{131}$ I treatment in ATC cells. Conclusion: These findings suggest that the inverse agonist of ERRy enhances the responsiveness of radioiodine therapy by modulating NIS function in ATC cells via the regulation of ERRy and the MAP kinase signaling pathway.

Received May 4, 2015; revision accepted Aug. 27, 2015. For correspondence or reprints contact either of the following:

Young Hyun Jeon, Leading-Edge Research Center for Drug Discovery and Development for Diabetes and Metabolic Disease, Kyungpook National University Hospital, 807 Hogukro, Bukgu, Daegu, South Korea, 702-210.

E-mail: jeon9014@gmail.com

Jaetae Lee, Department of Nuclear Medicine, Kyungpook National University School of Medicine, 50 Samduk-dong 2-ga, Chung Gu, Daegu, South Korea, 700-721.

E-mail: jaetae@knu.ac.k

Published online Sep. 3, 2015.

COPYRIGHT (C 2015 by the Society of Nuclear Medicine and Molecular Imaging, Inc.
Key Words: sodium iodide symporter (NIS); anaplastic thyroid cancer; estrogen-related receptor gamma (ERRy); radioiodide therapy; MAP kinase signaling

J Nucl Med 2015; 56:1690-1696

DOI: 10.2967/jnumed.115.160366

A naplastic thyroid cancer (ATC) is one of the most aggressive and lethal cancers known to affect humans $(1,2)$. ATC rapidly metastasizes from the thyroid glands to the lungs, bone, regional lymph nodes, and brain (3). This is in contrast to the benign nature of well-differentiated thyroid cancer, which accounts for most thyroid cancers. Treatment of ATC with surgery, radiotherapy, and chemotherapy alone or in combination has had little to no effect on patient survival (4-7). Consequently, the development of novel therapeutic approaches is urgently required.

The sodium iodide symporter (NIS) is a plasma membrane glycoprotein that mediates the active influx of iodide into cells (8). In thyroid cancer, endogenous NIS accommodates the widespread clinical application of radioiodine therapy, which has been shown over the years to be an effective method of eliminating malignant cells, with minimal adverse effects. Poorly differentiated cancer cells, including ATC cells, tend to exhibit a progressive dedifferentiation that leads to a decrease in levels of NIS $(9,10)$. This results in the inability of ATC cells to accumulate a high iodine concentration and hence their resistance to radionuclide therapy, which finally leads to poor prognosis (11). Thus, many attempts have been made to restore NIS function in ATC cells using methods such as gene delivery $(12,13)$ and epigenetic modulation with epigenome-modifying drugs (14), but few satisfactory outcomes have been achieved.

Estrogen-related receptors (ERR $\alpha, E R R \beta$, and ERR $\gamma)$ are constitutively active nuclear receptors that bear high levels of sequence identity to estrogen receptors (15). The ERR isoforms are primarily expressed in several organs, such as the heart, brain, kidney, pancreas, and liver $(16,17)$. Recently, studies have shown that ERR $\gamma$ is involved in metabolic diseases such as type 2 diabetes mellitus, alcohol-induced oxidative stress, liver injury, and microbial infection 
through impaired hepatic gluconeogenesis $(18,19)$, hepatic insulin signaling (20), and iron metabolism (21).

Crystal structure studies have demonstrated that ERRs are constitutively active without their ligand, and several small-molecule ligands can either activate or repress the functional activity of the ERRs (22). Among them, GSK5182 (a 4-hydroxy tamoxifen analog) is a selective inverse agonist of ERR $\gamma$ that does not interact with another nuclear receptor. Several lines of evidence have revealed that GSK5182 not only alleviates diabetes symptoms through the inhibition of hepatic gluconeogenesis in a PGC- $1 \alpha$ (peroxisome proliferator-activated receptor $\gamma$ coactivator- $1 \alpha$ )-dependent manner $(19,23)$ but also shows antimicrobial effects by the reduction of ERR $\gamma$-mediated hepcidin messenger RNA expression (21). Although the biologic effects of ERR $\gamma$ have been extensively investigated in various disease models, the role of ERR $\gamma$ in NIS function in ATC remains to be clearly determined.

Among various nuclear receptors the retinoic acid receptor, through its activator, retinoic acid, has been proven effective in restimulating iodide uptake in nonthyroidal cancer and thyroid cancer in vitro and in vivo $(24,25)$. We speculated that the ERR $\gamma$ of the nuclear receptor family might also be involved in controlling NIS function in thyroid cancer, similar to the biologic roles of RA and RAR on NIS function.

To explore the effects of ERR $\gamma$ on NIS function in ATC cells, we adopted GSK5182 as a specific inverse agonist of ERR $\gamma$ and investigated the changes in endogenous ERR $\gamma$ protein and MAP kinase signaling by this agent. The effects of GSK5182 on the functional activity and expression level of NIS protein in ATC cells were also assessed. Finally, the enhanced cytotoxic effects of ${ }^{131} \mathrm{I}$ from GSK5182 treatment were evaluated in ATC cells.

\section{MATERIALS AND METHODS}

\section{Cells}

Two ATC cell lines, BHT-101 and CAL-62, were purchased from Deutsche Sammlung von Mikroorganismen und Zellkulturen. Both cell lines were maintained in high-glucose Dulbecco modified Eagle medium supplemented with $10 \%$ fetal bovine serum and a $1 \%$ antibioticantimycotic (HyClone; GE Healthcare) at $37^{\circ} \mathrm{C}$ in a $5 \% \mathrm{CO}_{2}$ atmosphere.

\section{I Uptake Assay}

Cells were plated in 24-well plates for $24 \mathrm{~h}$ and then treated with GSK5182 synthesized by Daegu-Gyeongbuk Medical Innovation Foundation and prepared as a $100 \mathrm{mM}$ stock solution in dimethyl sulfoxide and stored at $-80^{\circ} \mathrm{C}$ for $24 \mathrm{~h}$. After aspiration of drugcontaining medium, the cells were washed with $1 \mathrm{~mL}$ of Hanks balanced salt solution (HBSS) and incubated with $500 \mu \mathrm{L}$ of HBSS containing $0.5 \%$ bovine serum albumin (bHBSS), $3.7 \mathrm{kBq}$ of carrierfree ${ }^{125} \mathrm{I}$ (Perkin-Elmer), and a $10 \mu \mathrm{mol} / \mathrm{L}$ solution of sodium iodide (specific activity of $740 \mathrm{MBq} / \mathrm{mmol}$ ) at $37^{\circ} \mathrm{C}$ for $30 \mathrm{~min}$. The cells were then washed twice with ice-cold bHBSS and were lysed with $500 \mu \mathrm{L}$ of $2 \%$ sodium dodecyl sulfate. The radioactivity was measured using a $\gamma$ counter (Cobra II; Canberra Packard, Packard Bioscience). The radioactivity of the cells was normalized using total protein concentrations determined by a bicinchoninic acid protein assay kit (Pierce Protein Biology). The cells were preincubated with $300 \mu \mathrm{M} \mathrm{KClO}_{4}$ (as a specific inhibitor for NIS) for $30 \mathrm{~min}$ to inhibit iodide uptake, followed by a ${ }^{125} \mathrm{I}$ uptake test as described above.

\section{Clonogenic Assay}

The cells were plated into 6-well plates and left for $24 \mathrm{~h}$. After treatment with $25 \mu \mathrm{M}$ GSK5182 for $24 \mathrm{~h}$, drug-containing medium was discarded and the cells were washed twice with phosphate-buffered saline (PBS). The medium was then replaced with Dulbecco modified Eagle medium in the presence or absence of $1,850 \mathrm{kBq}(50 \mu \mathrm{Ci})$ of ${ }^{131} \mathrm{I}$ (Korea Institute of Radiological and Medical Sciences) for $6 \mathrm{~h}$. The cells were washed with cold bHBSS and left in regular culture medium for the time corresponding to 6 doublings. Finally, the cells were fixed in $4 \%$ paraformaldehyde solution and stained with $0.05 \%$ crystal violet. Control and ${ }^{131} \mathrm{I}$ treated colonies with more than 50 cells were counted.

\section{Western Blot}

The cells were treated with or without GSK5182 for $24 \mathrm{~h}$ and washed twice with cold PBS and lysed with radioimmunoprecipitation assay buffer containing complete protease inhibitor cocktail (Roche). In case of plasma membrane protein for NIS, samples were prepared with a protein biotinylation kit (EZ-Link sulfo-NHS-biotin; Thermo Scientific) according to the manufacturer's instructions. Briefly, either untreated or treated cells were washed twice with ice-cold PBS/CM (PBS containing $0.1 \mathrm{mM}$ calcium chloride and $1 \mathrm{mM}$ magnesium chloride, pH 7.3) and incubated with EZ link NHS-sulfo-SS-biotin $\left(1 \mathrm{mg} / \mathrm{mL}\right.$ ) in PBS/CM for $30 \mathrm{~min}$ at $4{ }^{\circ} \mathrm{C}$. The reaction was quenched by 2 washes with cold $100 \mathrm{mM}$ glycine in PBS/CM and further incubation with $100 \mathrm{mM}$ glycine in $\mathrm{PBS} / \mathrm{CM}$ at $4{ }^{\circ} \mathrm{C}$ for $20 \mathrm{~min}$. The cells were then quickly washed 2 times with PBS/CM before lysis with radioimmunoprecipitation assay buffer containing a protease inhibitor cocktail and phosphatase inhibitors (Roche) for $1 \mathrm{~h}$ at $4^{\circ} \mathrm{C}$ with constant shaking. Lysates were centrifuged at $16,000 \mathrm{~g}$ for $30 \mathrm{~min}$ at $4^{\circ} \mathrm{C}$. A portion of the supernatant was used for total cell protein immunoblots. The remaining sample was used to obtain membrane protein by incubation with $100 \mu \mathrm{L}$ of streptavidin beads (Thermo Scientific) for $1 \mathrm{~h}$ at room temperature. The beads were washed 3 times with radioimmunoprecipitation assay buffer, and bound proteins were eluted with $50 \mu \mathrm{L}$ of Laemmli buffer (62.5 M Tris, pH 6.8; $20 \%$ glycerol; $2 \%$ sodium dodecyl sulfate; $5 \%$ b-mercaptoethanol; and $0.01 \%$ bromphenol blue) for $30 \mathrm{~min}$ at room temperature. Equal amounts of total and biotinylated plasma membrane protein were loaded in each lane and resolved by $4 \%-$ $12 \%$ gradient Bis-Tris gel (Invitrogen). Proteins were transferred to $0.2-\mu \mathrm{m}$ polyvinylidene fluoride membrane (Invitrogen). Membranes were incubated overnight at $4{ }^{\circ} \mathrm{C}$ with primary mouse monoclonal human NIS-specific antibody (dilution, 1:1,000, Thermo Scientific, catalog no. MS-1653-P1, clone: FP5A), followed by incubation with horseradish peroxidase-conjugated secondary antibody at room temperature. ECL-Plus (Amersham Pharmacia) was used to detect peroxidase activity according to the manufacturer's protocol.

Similarly, for other proteins also, equal amounts of protein were loaded in each lane and resolved by $4 \%-12 \%$ gradient Bis-Tris gel (Invitrogen). Proteins were transferred to a $0.2-\mu \mathrm{m}$ polyvinylidene fluoride membrane (Invitrogen). The membranes were incubated overnight at $4^{\circ} \mathrm{C}$ with primary antibodies and then with the appropriate horseradish peroxidaseconjugated secondary antibody at room temperature. ECL-Plus was used to detect peroxidase activity according to the manufacturer's protocol. Band densities were determined by ImageJ software.

\section{Statistical Analysis}

All data are expressed as mean $\pm \mathrm{SD}$, and statistical significance was determined using an unpaired Student test in Prism 5 (GraphPad). $P$ values of less than 0.05 were considered statistically significant.

\section{RESULTS}

\section{Enhanced Uptake in ATC Cells by Inverse Agonist of ERRy}

Treatment with GSK5182 significantly increased radioiodine uptake in both ATC cell lines in a dose-dependent fashion (Fig. 1A). The maximum relative increase in iodide uptake was 2.3- and 2.9-fold in the BHT-101 and CAL-62 cells, respectively, compared with the 


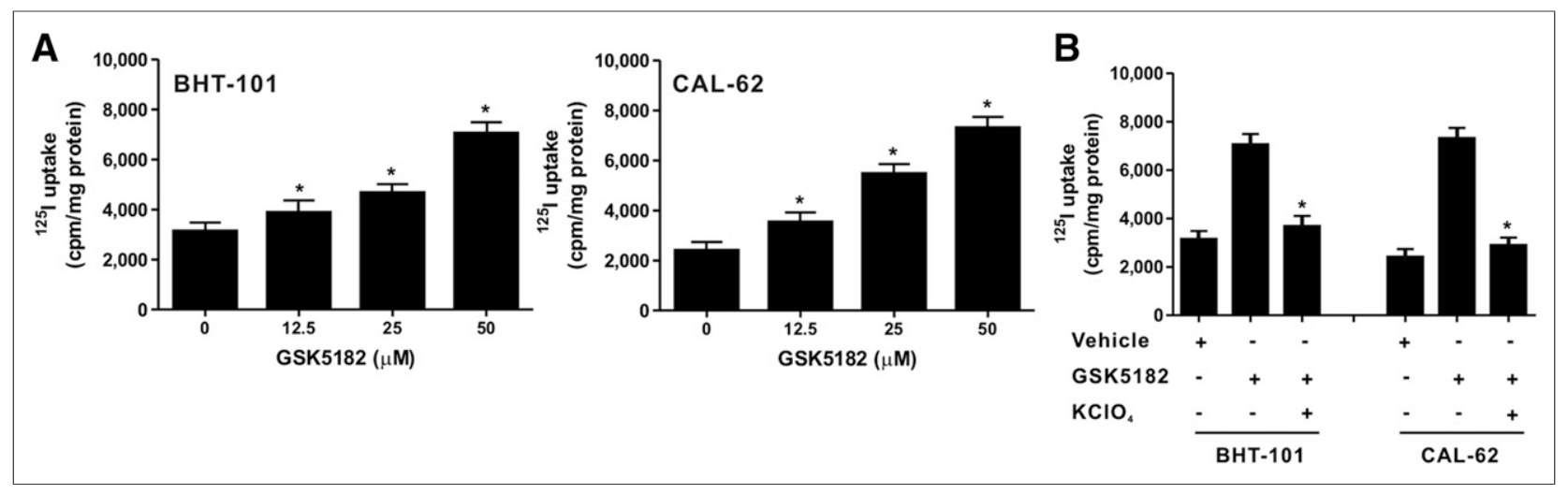

FIGURE 1. Effects of GSK5182 on kinetics of iodide uptake in the 2 ATC cell lines. (A) Each cell line was treated with various doses of GSK5182 for $24 \mathrm{~h}$, and dose-dependent increased iodide uptake was confirmed in both. (B) ${ }^{125}$ l uptake was inhibited in both cell lines by treatment with $300 \mu \mathrm{M}$ $\mathrm{KClO}_{4}$ for 30 min. ${ }^{\star} P<0.05$ when compared with GSK5182. Data are mean \pm SD of 3 samples per group.

vehicle group, which was obtained at a concentration of $50 \mu \mathrm{M}$ GSK5182. Accordingly, mainly $50 \mu \mathrm{M}$ GSK5182 was used for the further iodide uptake tests.

To examine whether the increased radioiodine uptake is related to modulation of NIS function by GSK5182, $\mathrm{KClO}_{4}$, a specific inhibitor of NIS, was coincubated with GSK5182-treated BHT-101 and CAL-62 cells and the change in radioiodine uptake levels was determined. The level of radioiodine uptake between vehicle-treated cells and combined vehicle and $\mathrm{KClO} 4$-treated cells did not differ between the two ATC cell lines, indicating that these lines have negligible NIS-mediated iodide uptake (Supplemental Fig. 1; supplemental materials are available at http://jnm.snmjournals.org). $\mathrm{KClO}_{4}$ completely blocked the enhanced radioiodine uptake in both types of GSK5182-treated cells (Fig. 1B), suggesting that the augmentation of iodide uptake is directly involved in the improved functional activity of NIS mediated by GSK5182.

We assessed the radioiodine uptake level in GSK5182-treated BHT-101 and CAL-62 cells according to different time points. Radioiodine uptake increased in both treated cell lines within as early as $2 \mathrm{~h}$ after treatment and peaked at $24 \mathrm{~h}$ (Supplemental Fig. 2).

\section{Downregulation of Endogenous ERRy by GSK5182 in} ATC Cells

To determine the effect of GSK5182 on ERR $\gamma$ protein levels in ATC cells, an immunoblotting analysis was conducted using an ERR $\gamma$ specific antibody. Both BHT-101 cells and CAL-62 cells showed endogenous expression of ERR $\gamma$ protein. Treatment with GSK5182 markedly reduced ERR $\gamma$ protein in both cells, with a relative decrease of 3.6-fold and 2.4-fold in the BHT-101 and CAL-62 cells, respectively (Fig. 2).

\section{Increase in Membrane-Localized NIS in ATC cells Through Activation of MAP Kinase Signaling}

A significant increase in phosphorylated MAP kinase levels, such as p44 and p42 extracellular signal-regulated kinase (ERK), was found in both ATC cell lines treated with GSK5182 (Fig. 3A). The relative increase in the phosphorylated forms of ERK1 and ERK2 was 1.6- and 2.1-fold, respectively, in BHT-101 cells. GSK5182 treatment resulted in 5.8- and 2.2-fold increases in phosphorylated p44 and p42 ERK, respectively, in CAL-62 cells and was completely inhibited by the selective mitogen-activated protein kinase kinase (MEK) inhibitor, PD98059 (Fig. 3B).

To determine the effect of GSK5182 on NIS protein status, we examined the change in levels of membranous and total NIS proteins collected from GSK5182-treated ATC cells using the plasma membrane biotinylation kit, followed by immunoblotting with NIS-specific antibody. As illustrated in Figure 4A, GSK5182 induced drastic increases in plasma membrane-localized NIS

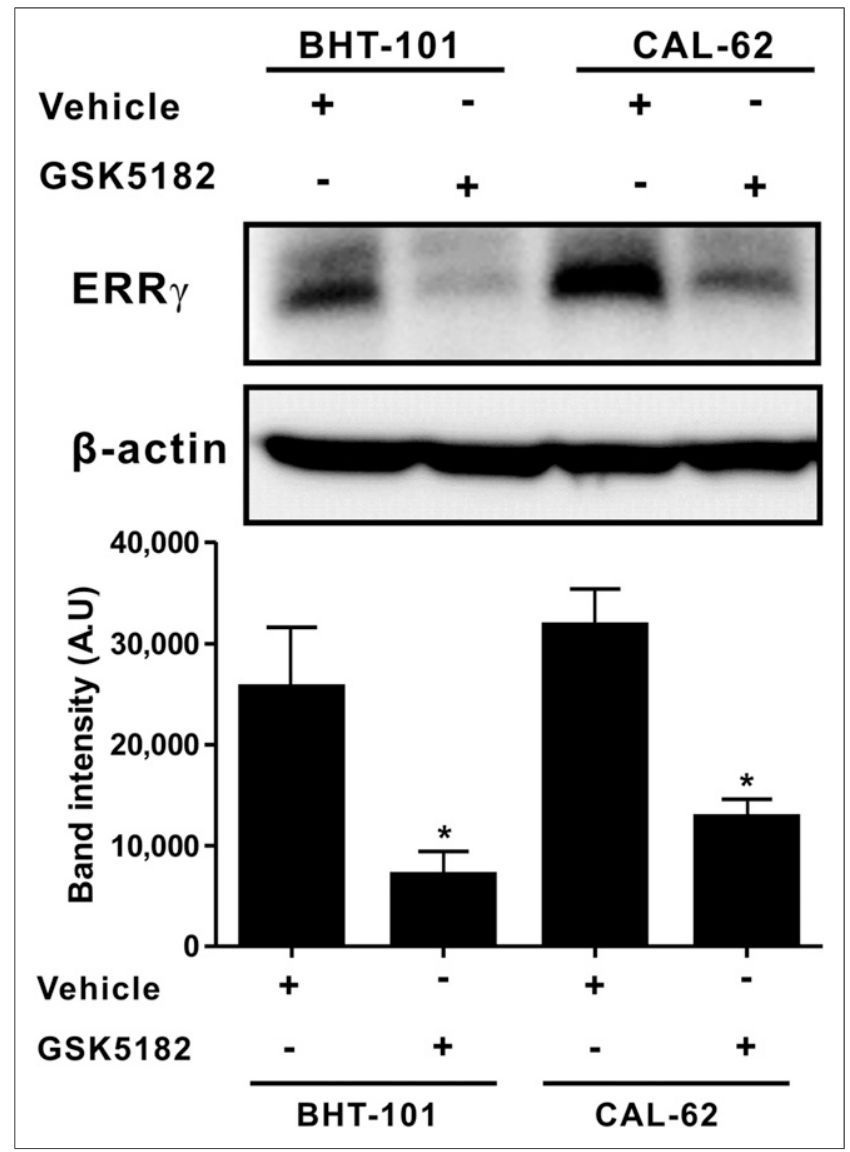

FIGURE 2. Effects of GSK5182 on endogenous ERRy protein in ATC cells. Endogenously expressed ERRy protein levels in ATC cells were decreased by GSK5182. Each cell line was treated with $50 \mu \mathrm{M}$ GSK5182 for $24 \mathrm{~h}$, and immunoblotting was done with ERRy-specific antibody. Graphs depicts results of quantitative analysis of ERRy protein levels by calibrated densitometer. ${ }^{*} P<0.05$ when compared with untreated cells. Data are mean $\pm \mathrm{SD}$ of 3 samples per group, expressed in arbitrary units. 


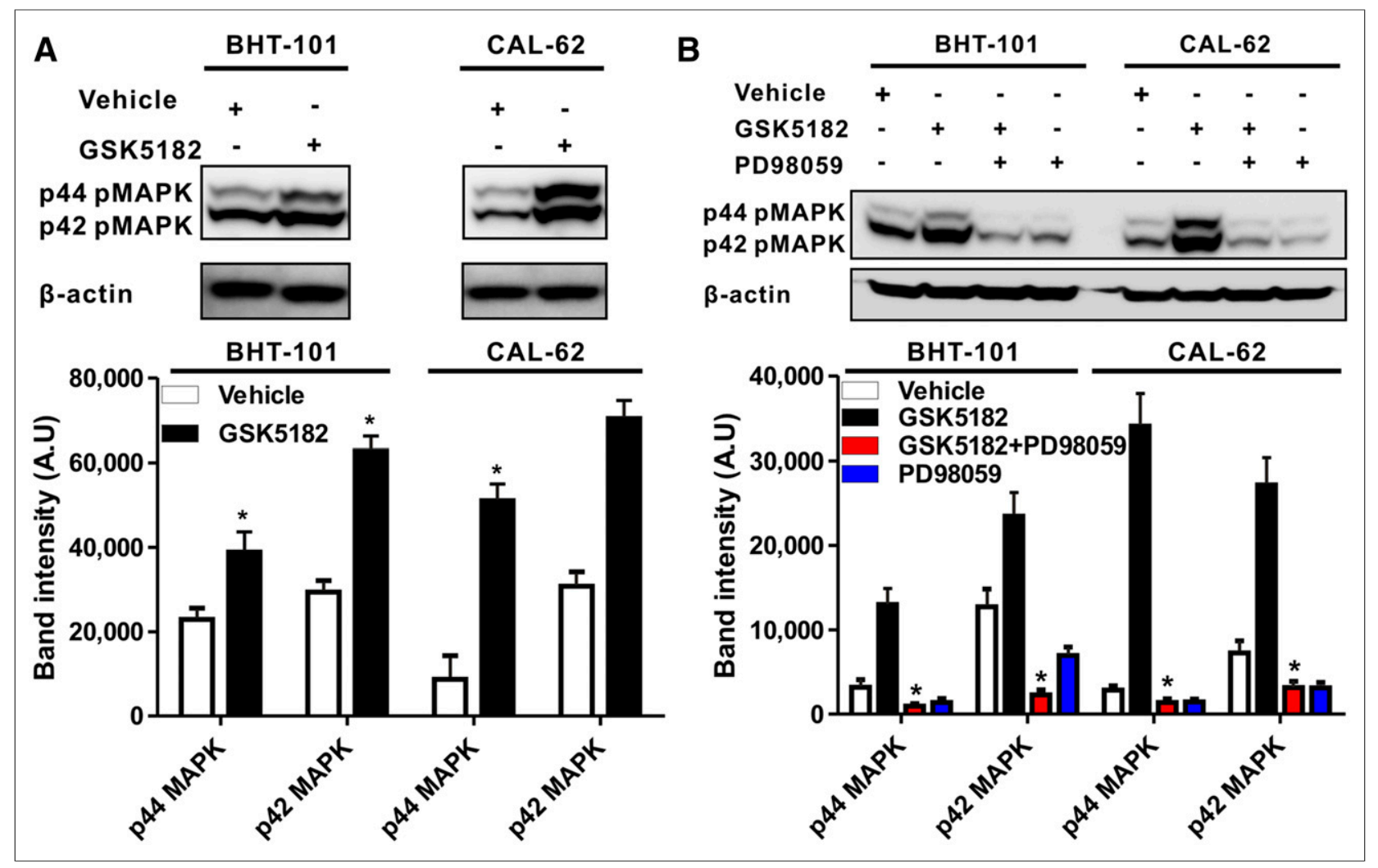

FIGURE 3. GSK5182-induced MAP kinase activation in ATC cells. (A) Activation of MAP kinase signaling in ATC cells by GSK5182. Each cell line was incubated with $50 \mu \mathrm{M}$ GSK5182 for $24 \mathrm{~h}$, and phosphorylated ERK1/2 levels were determined with phospho-p44/42 specific antibody. Graphs depict quantitative analysis of phosphorylated p44/42-ERK levels by scanning densitometry. ${ }^{*} P<0.05$ when compared with untreated cells. Data are mean \pm SD of 3 samples per group, expressed in arbitrary units. (B) Reversal of activated MAK kinase signaling by PD98059. Cells were cotreated with both $20 \mu \mathrm{M}$ PD98059 and GSK5182, and phosphorylated ERK1/2 levels were determined with immunoblotting. Graphs depict quantitative analysis of phosphorylated p44/42-ERK levels by scanning densitometry after cotreatment with both $20 \mu \mathrm{M}$ PD98059 and GSK5182. ${ }^{\star} P<0.05$ when compared with GSK5182 cells. Data are mean \pm SD of 3 samples per group, expressed in arbitrary units.

protein, with an immature form in both ATC cell lines, compared with control cells. Quantitative analysis of the band intensity revealed 3.8- and 6.0-fold increases in membrane NIS protein in the BHT-101 and CAL-62 cells, respectively (Supplemental Fig. 3). However, there was no significant change in the levels of NIS protein in total cell lysates of untreated and GSK5182-treated ATCs. A radioiodine uptake test with PD98059 treatment demonstrated that the GSK5182-induced iodide uptake reverted to the basal level in both ATC cell lines, whereas incubation of cells with PD98059 alone did not affect iodide uptake (Fig. 4B).

\section{Amelioration of ${ }^{131}$ I-Mediated Cytotoxicity by GSK5182 in ATCs}

As shown in Figure 5 and Supplemental Figure 4, clonogenic assay with ${ }^{131}$ I demonstrated minimal cytotoxic effects in the ATC cells treated with either GSK5182 or ${ }^{131}$ I alone. The relative colonyforming ability in CAL-62 cells for the ${ }^{131}$ I and GSK5182 groups was $92.9 \% \pm 5.8 \%$ and $94.5 \% \pm 10.8 \%$, respectively. Similar to CAL-62 cells, BHT-101 cells had a relative colony-forming ability of $95.2 \% \pm 4.2 \%$ and $93.2 \% \pm 5.5 \%$ in the ${ }^{131} \mathrm{I}$ and GSK5182 groups, respectively. However, the combination of GSK5182 with ${ }^{131}$ I markedly reduced the colony-forming ability to approximately $58.5 \% \pm 7.4 \%$ and $72.8 \% \pm 2.4 \%$ in CAL-62 and BHT-101, respectively.

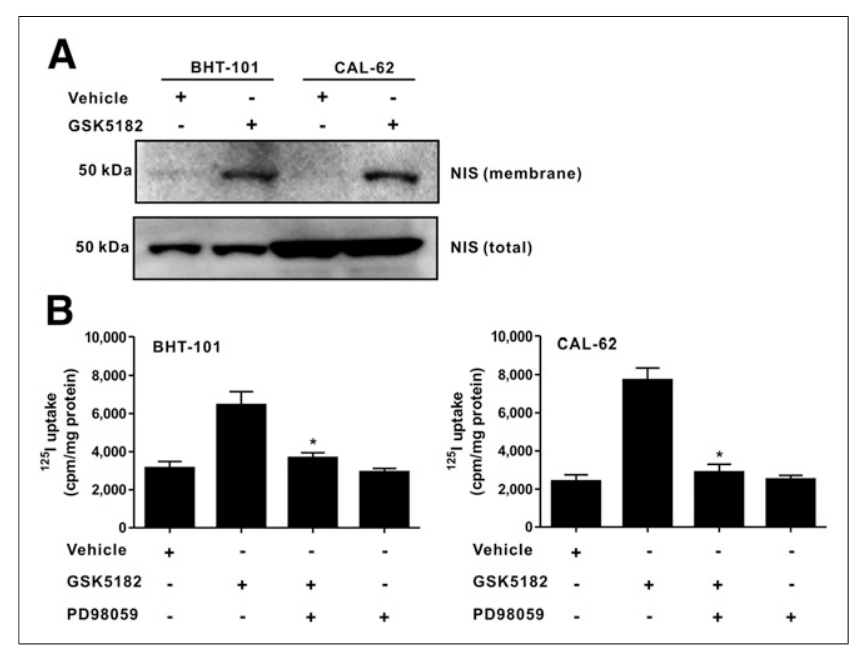

FIGURE 4. Enhancement of membrane-localized NIS protein level by GSK5182. (A) Detection of membrane and total NIS protein. Membranous fraction of cells treated with or without GSK5182 was collected and immunoblotting analysis was done with NIS-specific antibody. (B) Inhibition of increased iodide uptake by PD98059 in GSK5182-treated ATC cells. Cells were cotreated with both $20 \mu \mathrm{M}$ PD98059 and GSK5182, and iodide uptake levels were determined. ${ }^{\star} P<0.05$ when compared with GSK5182. Data are mean \pm SD of 3 samples per group. 


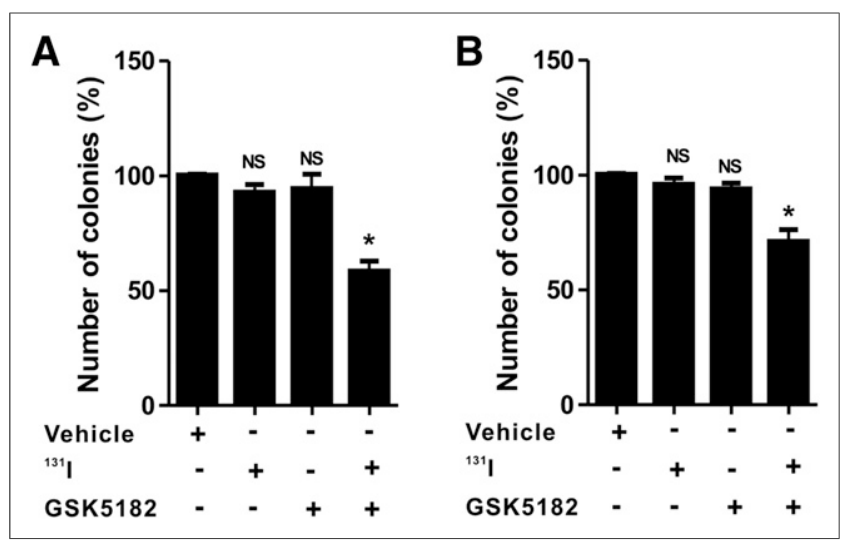

FIGURE 5. Increased cytotoxicity of ${ }^{131}$ I by GSK5182 against ATC cells. CAL-62 (A) and BHT-101 (B) cells were pretreated with or without GSK5182 before day 1 . After being washed, they were further coincubated with $1,850 \mathrm{kBq}(50 \mu \mathrm{Ci})$ of ${ }^{131}$ I for $6 \mathrm{~h}$. Graphs depict percentage of colonies after ${ }^{131}$ I treatment. ${ }^{*} P<0.05$. NS $=$ not significant when compared with untreated cells. Data are mean \pm SD of 3 samples per group.

\section{DISCUSSION}

We have demonstrated that GSK5182, an inverse agonist of ERR $\gamma$, reduces the endogenous ERR $\gamma$ protein level in ATC cells and leads to the activation of MAP kinase signaling (Fig. 6). Importantly, treatment with GSK5182 enhances radioiodine uptake in ATC cells in a dose- and time-dependent fashion. Both the GSK5182-induced ERK1/2 activation and the increase in radioiodine uptake are completely inhibited by PD98059, a selective MEK inhibitor. Further examination showed that GSK5182 increases NIS plasma membrane protein without affecting levels of total NIS protein. Furthermore, the enhancement of NIS function modulated by GSK5182 leads to increased susceptibility of ATC cells to radioiodine therapy.

Recently, many studies have shown interesting therapeutic outcomes from GSK5182-mediated ERR $\gamma$ regulation in various

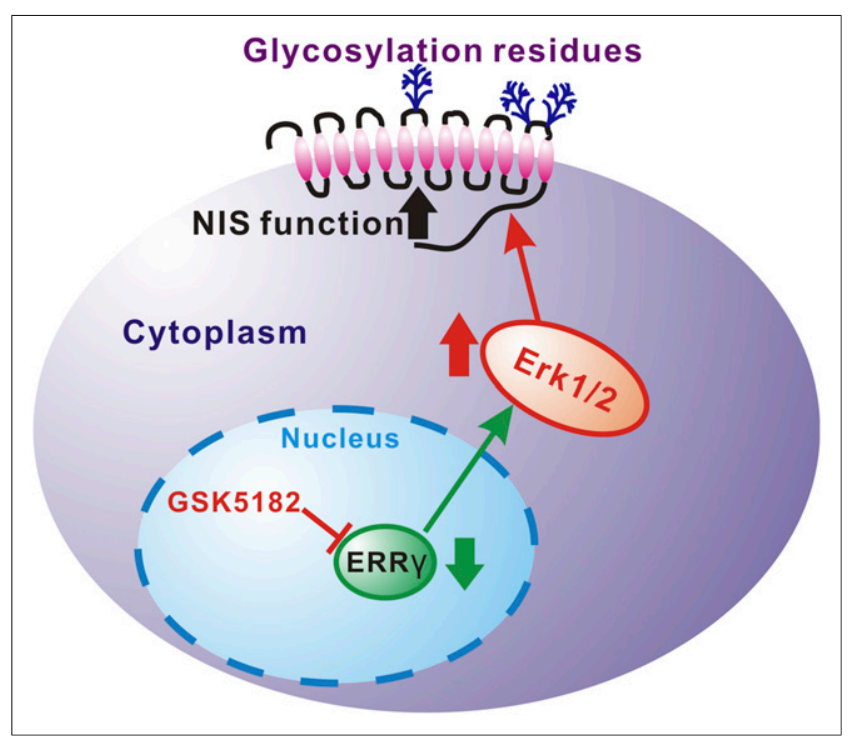

FIGURE 6. Proposed mechanism of GSK5182-induced modulation of NIS function in ATC cells through MAP kinase pathway. metabolic and cardiac diseases $(18,20,21,26)$, but no studies have unraveled the involvement of GSK5182-modified ERR $\gamma$ in regulating NIS function in thyroid cancer, especially ATC. In an attempt to determine the effects of GSK5182 on radioiodine uptake in ATC cells, we selected 2 different ATC cells, BHT-101 and CAL-62, which have characteristic mutations of the K-RAS and BRAF genes, respectively. These cell lines have been used to evaluate the therapeutic efficacy of several drugs in inducing cytotoxic effects directly as well as modulating NIS function $(14,27)$. Interestingly, the incubation of ATC cells with GSK5182 led to doseand time-dependent increases in radioiodine uptake, but negligible uptake was shown in vehicle-treated cells. From these findings, we questioned whether the increased iodide uptake was associated with modulated NIS function. Thus, an iodide uptake test was further performed by introducing a specific inhibitor of NIS protein, potassium perchlorate $\left(\mathrm{KClO}_{4}\right)$, to GSK5182treated cells. $\mathrm{KClO}_{4}$ has been widely used to validate the functional activity of NIS protein (8). Iodide uptake analysis with $\mathrm{KClO}_{4}$ clearly showed the inhibition of enhanced radioiodine uptake to basal level, revealing that increased iodide uptake is related to the GSK5182-induced modulation of NIS function. Next, we speculated that the enhanced iodide uptake might be involved in a change in endogenous ERR $\gamma$ protein by GSK5182. We observed that both ATC cell lines exhibited a substantial and similar level of expression of ERR $\gamma$ protein, and treatment with GSK5182 significantly reduced the ERR $\gamma$ protein level. Taken together, these results suggest that the GSK5182induced reduction of endogenous ERR $\gamma$ protein may affect NIS function and finally lead to increased radioiodine uptake in ATC cells.

MAP kinases are evolutionarily conserved enzymes that transfer signals from cell surface receptors to critical intracellular molecules (28). Among the MAP kinases, p38 MAP kinase has been shown to have a role in maintaining normal basal expression of serotonin transporters (29) and improving the activation of norepinephrine transporters (30). Knostman et al. reported that PI3K activation induces underglycosylated intracellular NIS protein expression in breast cancer (MCF-7) cells and leads to iodide uptake ability (31). Another report has shown that MEK inhibitor decreases NIS protein levels through lysosome-mediated NIS degradation in human breast cancer cells, suggesting that MEK activation has as an important role in maintaining NIS protein stability in human breast cancers (32). More recently, Lee et al. (12) reported that activation of protein kinase C signaling with phorbol 12-myristate 13-acetate down-regulated iodide uptake in NIS-expressing nonthyroidal cancer cells and that EGF-mediated MAP kinase activation enhanced radioiodine uptake. On the basis of these studies, it was presumed that the enhanced radioiodine uptake of ATC cells might be linked to upregulation of these kinase pathways. From an immunoblotting analysis with phosphor-ERK1/2-specific antibody, we found that ERK signaling is constitutively active in both ATC cell lines and that treatment with GSK5182 increases the phosphorylated MAP kinase level. Conversely, PD98059, a selective inhibitor of MAP kinase, inhibits the increased phosphorylation of ERK1/2 MAP kinase as well as the iodide uptake induced by GSK5182. It would therefore be reasonable to postulate that the enhanced radioiodine uptake of ATC cells may be associated with the activation of MAP kinase signaling that results from the GSK5182-mediated reduction of ERR $\gamma$ protein.

The total amount of NIS protein, as well as plasma-membranelocalized NIS protein, is crucial to the capacity of thyroid cells to transport iodide. Even though sufficient expression of NIS protein is 
observed in thyroid cancer cells, intracellular retention of NIS prevents iodide uptake from being sufficient to generate an effective radioiodine therapy. The NIS transporter needs to be translocated to the plasma membrane to be fully functionalized in thyroid cells. Thyroid-stimulating hormone is needed to generate membrane retention of NIS, and its depletion leads to an acute decrease in iodide uptake in thyroid cells (33). Furthermore, breast cancer cells with sufficient endogenous expression of NIS had a poor ability to take up iodide, partially resulting from intracellular localization of NIS protein $(24,34)$.

On the basis of these reports, we speculated that both the downregulation of ERR $\gamma$ protein level and activation of MAP kinase by GSK5182 may affect total and plasma membrane NIS protein status. We could detect the endogenously expressed total NIS protein without an increase in membrane NIS protein in vehicle-treated cells. Although several reports have demonstrated that ATC cells do not express NIS protein (35), we could evidently detect NIS protein expression in both ATC cell lines in this study. The discrepancy may be due to the development of anti-NIS-specific antibody with high sensitivity and specificity as well as better experimental conditions. Surprisingly, biotinylation experiments with GSK5182treated ATC cells revealed an increase in immature NIS protein at the plasma membrane. But there was no change in the NIS protein in total cell lysate protein. The exact mechanism responsible for the GSK5182-mediated increase in iodide transport is not yet clear. Because the general posttranscriptional mechanism of both the localization and the function of many transporters includes the glycosylation and phosphorylation processes (36), similar processes may be involved in NIS regulation.

Successful radioiodine therapy against thyroid cancer depends mainly on effective accumulation of radioiodine. It would be reasonable to postulate that the enhanced radioiodine uptake brought about by GSK5182 may induce cytotoxic effects against ATC cells. Notably, in our study GSK5182 treatment was sufficient to enhance the killing effect of therapeutic radioiodine on ATC cells, but treatment with ${ }^{131}$ I alone could not induce sufficient cytotoxic effects on ATC cells. These findings suggest that ERR $\gamma$-modulated NIS function can be a reasonable approach to achieving a radioiodine accumulation that allows for acceptable therapeutic outcomes with clinically used doses of ${ }^{131} \mathrm{I}$. Further preclinical investigations using nuclear medicine imaging instruments such as SPECT or PET are required to evaluate the effects of GSK5182-mediated MAP kinase activation on the efficacy of radioiodine accumulation in ATC cancer in living mice.

\section{CONCLUSION}

Our findings suggest that ERR $\gamma$-regulated MAP kinase pathways play an important role in regulating NIS function. The findings also suggest that it is realistically feasible to appropriately regulate these signaling pathways to enhance NIS function and enable radioiodine therapy, thus allowing new therapeutic strategies for ATC to be uncovered. In the future, the ERR $\gamma$ inverse agonist can be introduced as an adjuvant in the development of new therapeutic protocols for patients with ATCs. Our current study guarantees further investigations for new drug candidates or GSK5182 derivatives to regulate ERR $\gamma$ activity and mediate a more effective reinduction of radioiodine uptake in ATC cells. Because the lack of iodide organification limits effective radioiodine therapy in ATC cells, further study is needed to learn the effects of GSK5182 on iodide organification in ATC cells.

\section{DISCLOSURE}

The costs of publication of this article were defrayed in part by the payment of page charges. Therefore, and solely to indicate this fact, this article is hereby marked "advertisement" in accordance with 18 USC section 1734. This work was supported by National Research Foundation of Korea (NRF) grants funded by the Korean government (MSIP) (2009-0078222 and 2009-0078234); a grant from the Korea Health Technology R\&D Project, Ministry of Health and Welfare (HI11C1300); the National Nuclear R\&D Program through the NRF funded by the Ministry of Education, Science and Technology (2012M2A2A7014020); a grant from the Medical Cluster R\&D Support Project through the Daegu-Gyengbuk Medical Innovation Foundation (DGMIF) funded by the Ministry of Health and Welfare (HT13C0002); the BK21 PlusKNU Biomedical Convergence Program, Department of Biomedical Science, Kyungpook National University; an NRF grant funded by the Korean government (MSIP) (2014R1A1A1003323); a grant from the Korea Health Technology R\&D Project through the Korea Health Industry Development Institute (KHIDI) funded by the Ministry of Health and Welfare (HI15C0001); and an NRF grant funded by the Korean government (MSIP) (NRF-2015M2A2A7A01045177). No other potential conflict of interest relevant to this article was reported.

\section{REFERENCES}

1. Fassnacht M, Kreissl MC, Weismann D, Allolio B. New targets and therapeutic approaches for endocrine malignancies. Pharmacol Ther. 2009;123:117-141.

2. Nagaiah G, Hossain A, Mooney CJ, Parmentier J, Remick SC. Anaplastic thyroid cancer: a review of epidemiology, pathogenesis, and treatment. J Oncol. 2011;2011:542358.

3. De Falco V, Guarino V, Avilla E, et al. Biological role and potential therapeutic targeting of the chemokine receptor CXCR4 in undifferentiated thyroid cancer. Cancer Res. 2007;67:11821-11829.

4. Busnardo B, Daniele O, Pelizzo MR, et al. A multimodality therapeutic approach in anaplastic thyroid carcinoma: study on 39 patients. J Endocrinol Invest. 2000;23:755-761.

5. Aldinger KA, Samaan NA, Ibanez M, Hill CS Jr. Anaplastic carcinoma of the thyroid: a review of 84 cases of spindle and giant cell carcinoma of the thyroid. Cancer. 1978;41:2267-2275.

6. Kim JH, Leeper RD. Treatment of locally advanced thyroid carcinoma with combination doxorubicin and radiation therapy. Cancer. 1987;60:2372-2375.

7. Tennvall J, Lundell G, Wahlberg P, et al. Anaplastic thyroid carcinoma: three protocols combining doxorubicin, hyperfractionated radiotherapy and surgery. Br J Cancer. 2002;86:1848-1853.

8. Chung JK. Sodium iodide symporter: its role in nuclear medicine. J Nucl Med. 2002;43:1188-1200.

9. Rosai J. Poorly differentiated thyroid carcinoma: introduction to the issue, its landmarks, and clinical impact. Endocr Pathol. 2004;15:293-296.

10. Patel KN, Shaha AR. Poorly differentiated and anaplastic thyroid cancer. Cancer Control. 2006;13:119-128.

11. Kogai T, Taki K, Brent GA. Enhancement of sodium/iodide symporter expression in thyroid and breast cancer. Endocr Relat Cancer. 2006;13:797-826.

12. Lee YJ, Chung JK, Kang JH, Jeong JM, Lee DS, Lee MC. Wild-type p53 enhances the cytotoxic effect of radionuclide gene therapy using sodium iodide symporter in a murine anaplastic thyroid cancer model. Eur J Nucl Med Mol Imaging. 2010;37:235-241.

13. Lee YJ, Chung JK, Shin JH, et al. In vitro and in vivo properties of a human anaplastic thyroid carcinoma cell line transfected with the sodium iodide symporter gene. Thyroid. 2004;14:889-895.

14. Pugliese M, Fortunati N, Germano A, et al. Histone deacetylase inhibition affects sodium iodide symporter expression and induces ${ }^{131} \mathrm{I}$ cytotoxicity in anaplastic thyroid cancer cells. Thyroid. 2013;23:838-846.

15. Tremblay AM, Giguere V. The NR3B subgroup: an ovERRview. Nucl Recept Signal. 2007;5:e009.

16. Heard DJ, Norby PL, Holloway J, Vissing H. Human ERRgamma, a third member of the estrogen receptor-related receptor (ERR) subfamily of orphan nuclear receptors: tissue-specific isoforms are expressed during development and in the adult. Mol Endocrinol. 2000;14:382-392. 
17. Hong H, Yang L, Stallcup MR. Hormone-independent transcriptional activation and coactivator binding by novel orphan nuclear receptor ERR3. J Biol Chem. 1999;274:22618-22626.

18. Kim DK, Gang GT, Ryu D, et al. Inverse agonist of nuclear receptor ERRgamma mediates antidiabetic effect through inhibition of hepatic gluconeogenesis. $\mathrm{Di}$ abetes. 2013;62:3093-3102.

19. Kim DK, Ryu D, Koh M, et al. Orphan nuclear receptor estrogen-related receptor gamma (ERRgamma) is key regulator of hepatic gluconeogenesis. J Biol Chem. 2012;287:21628-21639.

20. Kim DK, Kim JR, Koh M, et al. Estrogen-related receptor gamma (ERRgamma) is a novel transcriptional regulator of phosphatidic acid phosphatase, LIPIN1, and inhibits hepatic insulin signaling. J Biol Chem. 2011;286:38035-38042.

21. Kim DK, Jeong JH, Lee JM, et al. Inverse agonist of estrogen-related receptor gamma controls Salmonella typhimurium infection by modulating host iron homeostasis. Nat Med. 2014;20:419-424.

22. Giguère V. Transcriptional control of energy homeostasis by the estrogen-related receptors. Endocr Rev. 2008;29:677-696.

23. Chao EY, Collins JL, Gaillard S, et al. Structure-guided synthesis of tamoxifen analogs with improved selectivity for the orphan ERRgamma. Bioorg Med Chem Lett. 2006;16:821-824.

24. Kogai T, Schultz JJ, Johnson LS, Huang M, Brent GA. Retinoic acid induces sodium/iodide symporter gene expression and radioiodide uptake in the MCF-7 breast cancer cell line. Proc Natl Acad Sci USA. 2000;97:8519-8524.

25. Kogai T, Kanamoto Y, Che LH, et al. Systemic retinoic acid treatment induces sodium/iodide symporter expression and radioiodide uptake in mouse breast cancer models. Cancer Res. 2004;64:415-422.

26. Kwon DH, Eom GH, Kee HJ, et al. Estrogen-related receptor gamma induces cardiac hypertrophy by activating GATA4. J Mol Cell Cardiol. 2013;65:88-97.
27. Catalano MG, Fortunati N, Pugliese M, et al. Histone deacetylase inhibition modulates E-cadherin expression and suppresses migration and invasion of anaplastic thyroid cancer cells. J Clin Endocrinol Metab. 2012;97:E1150-E1159.

28. Chang L, Karin M. Mammalian MAP kinase signalling cascades. Nature. 2001;410:37-40.

29. Samuvel DJ, Jayanthi LD, Bhat NR, Ramamoorthy S. A role for p38 mitogenactivated protein kinase in the regulation of the serotonin transporter: evidence for distinct cellular mechanisms involved in transporter surface expression. J Neurosci. 2005;25:29-41.

30. Apparsundaram S, Sung U, Price RD, Blakely RD. Trafficking-dependent and -independent pathways of neurotransmitter transporter regulation differentially involving p38 mitogen-activated protein kinase revealed in studies of insulin modulation of norepinephrine transport in SK-N-SH cells. J Pharmacol Exp Ther. 2001;299:666-677.

31. Knostman KA, McCubrey JA, Morrison CD, Zhang Z, Capen CC, Jhiang SM. $\mathrm{PI} 3 \mathrm{~K}$ activation is associated with intracellular sodium/iodide symporter protein expression in breast cancer. BMC Cancer. 2007;7:137.

32. Zhang Z, Beyer S, Jhiang SM. MEK inhibition leads to lysosome-mediated $\mathrm{Na}+/ \mathrm{I}-$ symporter protein degradation in human breast cancer cells. Endocr Relat Cancer. 2013;20:241-250.

33. Riedel C, Levy O, Carrasco N. Post-transcriptional regulation of the sodium/ iodide symporter by thyrotropin. J Biol Chem. 2001;276:21458-21463.

34. Arturi F, Ferretti E, Presta I, et al. Regulation of iodide uptake and sodium/iodide symporter expression in the mcf-7 human breast cancer cell line. J Clin Endocrinol Metab. 2005;90:2321-2326.

35. Spitzweg C, Harrington KJ, Pinke LA, Vile RG, Morris JC. Clinical review 132: the sodium iodide symporter and its potential role in cancer therapy. J Clin Endocrinol Metab. 2001;86:3327-3335.

36. Ramamoorthy S, Blakely RD. Phosphorylation and sequestration of serotonin transporters differentially modulated by psychostimulants. Science. 1999;285:763-766. 